\title{
Performance of VoIP with DCCP for satellite links
}

\author{
Golam Sarwar*,,$\ddagger$, Roksana Boreli*, \\ * National ICT Australia Ltd, Australia, \\ $\dagger$ University of NSW, Kensington, Australia, \\ $\ddagger$ 7-ip, Eveleigh, Sydney, Australia, \\ forename.surname@nicta.com.au
}

\author{
Emmanuel Lochin \\ CNRS ; LAAS ; \\ 7 avenue du colonel Roche, F-31077 Toulouse, France \\ ${ }^{2}$ Université de Toulouse ; \\ UPS, INSA, INP, ISAE ; LAAS ; \\ F-31077 Toulouse, France
}

\begin{abstract}
We present experimental results for the performance of selected voice codecs using the Datagram Congestion Control Protocol (DCCP) with TCP-Friendly Rate Control (TFRC) congestion control mechanism over a satellite link. We evaluate the performance of both constant and variable data rate speech codecs (G.729, G.711 and Speex) for a number of simultaneous calls, using the ITU E-model and identify problem areas and potential for improvement. Our experiments are done on a commercial satellite service using a data stream generated by a VoIP application, configured with selected voice codecs and using the DCCP/CCID4 Linux implementation. We analyse the sources of packet losses which are a main contributor to reduced voice quality when using CCID4 and additionally analyse the effect of jitter which is one of the crucial parameters contributing to VoIP quality and has, to the best of our knowledge, not been considered previously in the published DCCP performance results. We propose modifications to the CCID4 algorithm and demonstrate how these improve the VoIP performance, without the need for additional link information other than what is already monitored by CCID4 (which is the case for Quick Start). We also demonstrate the fairness of the proposed modifications to other flows. We identify the additional benefit of DCCP when used in VoIP admission control mechanisms and draw conclusions about the advantages and disadvantages of the proposed DCCP/ CCID4 congestion control mechanism for use with VoIP applications.
\end{abstract}

\section{INTRODUCTION}

Voice over IP (VoIP) has become a well established technology with a large number of operators offering services and an ever growing number of end users. A large proportion of VoIP services use the public Internet, rather than a globally reserved bandwidth. This presents a problem both for the VoIP quality and the congestion of public Internet, as VoIP most commonly uses the UDP protocol which has no congestion control and has no concept of fairness to other flows which share the same networks.

To bridge the gap between UDP and TCP, which is a reliable transport protocol and is not suitable for real time traffic, a new transport protocol for multimedia applications, Datagram Congestion Control Protocol (DCCP), has been proposed by IETF [1]. The main driver for having congestion control in an unreliable transport protocol was fairness to TCP traffic, which constitutes majority of the traffic on any Internet link. DCCP includes multiple congestion control algorithms identified by the Congestion Control ID (CCID), so that the application not needing reliable transport can select the appropriate congestion control method. CCID3[2] and its small packet variant CCID4[4] rely on the TCP-Friendly Rate Control (TFRC) algorithm which is suitable for traffic with smooth changes in sending rates, such as telephony or video streaming. The TFRC is based on the TCP throughput equation [3] and is therefore shown to be reasonably fair when competing with TCP flows. CCID3 is more suitable for streaming applications while CCID4 has been designed for applications with small packets and is primarily applicable to VoIP.

In geographically large countries with sparse population outside of the main centres like Australia, US or Canada and also in countries with a quickly growing infrastructure like India, there has been a number of new satellite network deployments in recent times [4]-[6]. These networks have an increasing amount of multimedia and real time traffic and need to be considered in developing and evaluating new protocols like DCCP.

In this paper, we present results of an experimental evaluation of the performance of selected voice codecs which use DCCP/CCID4 with TFRC congestion control over the IPSTAR satellite network [6] which is operational in Australia and a large number of other countries in Asia. To the best of our knowledge, this study is the first one presenting results from live satellite network performance measurements of a real VoIP data stream using DCCP. We measure the losses and the variation in the received voice packet delay as perceived by the VoIP application and evaluate the VoIP quality under different conditions of network load using the ITU E-model [16]. We also evaluate fairness to competing TCP traffic sharing the same network. To mitigate the packet loss resulting from DCCP/CCID4 congestion control, we propose a modification to the CCID4 algorithm, which, compared to an alternative proposal QuickStart [11], does not require any additional information in regards to the link rate from the receiver. We demonstrate that the modification results in a significantly improved VoIP quality compared to the original CCID4, while still preserving the farness advantage to other flows that CCID4 has over UDP.

The rest of the paper is structured as follows. Section II provides an overview of the related work and a description of the DCCP congestion control mechanism used for VoIP. Section III presents the experimental setup for live satellite tests. The following sections present experimental results, analysis of the results and our proposed modifications to the CCID4 protocol. In Section VI we present evaluation of the VoIP quality for different protocols. Section VII presents 
conclusions and a discussion of future work.

\section{TFRC AND CONGESTION CONTROL FOR VOIP}

This section presents an overview of the TFRC congestion control mechanism and a summary of related work.

DCCP/CCID3 [2] and DCCP/CCID4 [4] use TFRC [3] congestion control. In the TFRC congestion control mechanism, based on the monitored network conditions, the appropriate sending rate is computed by a combination of mechanisms in the sender and the receiver. Sender regulates the transmitted rate based on the received feedback messages which include the measured received rate, delay and an approximation of the packet loss rate. TFRC congestion control includes, similar to TCP congestion control, a slow start phase and a congestion avoidance phase. In slow start, before the sender has received any receiver feedback, the senders transmit rate $X$ is set to one packet per second [2].

This is also the minimum rate maintained by the CCID3/CCID4 sender at any time [2]. After the receiver feedback is available, the senders initial rate is calculated as equation 1

$$
X=\frac{\min (4 \cdot s, \max (2 \cdot s, 4380))}{R T T}
$$

Where:

- $R T T$ is the estimated round trip time in seconds

- $s$ the packet mean size in bytes.

During the reminder of the slow start phase, the sender rate is approximately doubled with every received feedback, as per equation 2

$$
X=\max \left(2 \cdot X, 2 \cdot X_{\text {recv }}\right)
$$

Where $X_{r e c}$ is the received reported rate in bytes/second.

When the receiver reports the first error, TFRC enters the congestion avoidance phase, which uses equation 3 approximating the transmitted rate to what would be an equivalent rate of TCP under the same network conditions.

$$
X=s \cdot f(p, R T T)
$$

Where,

$$
f(p, R T T)=\frac{1}{\left(R T T \cdot \sqrt{\frac{p \cdot 2}{3}}+R T O \cdot \sqrt{\frac{p \cdot 27}{8}} \cdot p \cdot\left(1+32 \cdot p^{2}\right)\right)}
$$

Where,

- $p$ is the loss event rate, between 0 and 1.0, to represent the number of loss events as a fraction of the number of packets transmitted;

- $R T O$ is the TCP retransmission timeout value in seconds.

CCID4 [4] differs from CCID3 only in the congestion avoidance phase. To calculate the sending rate, in place of the packet size $s$ in formula (6), CCID4 uses a fixed packet size of 1460 bytes modified by a header correction factor, resulting in the following equation 5

$$
X=1460 \cdot f(p, R T T) \cdot \frac{s}{s+o h}
$$

Where,

- oh is the size of protocol overhead in Bytes.

This change to the rate control ensures that the formula based rate is fair to both TCP and DCCP traffic: the rate is equivalent to the rate of TCP which would most commonly have larger packet sizes than DCCP traffic which consists of small VoIP packets; the calculated rate is reduced appropriately by the header correction factor, as the header size is significant compared to the size of the VoIP application payload.

In previous work, the performance of VoIP with DCCP/CCID4 protocol over satellite links has been studied in [10], [11], using ns-2 [14] simulation. The authors propose the use of Quick Start [15] and Fast Restart [16] mechanisms and show that these methods provide only a partial improvement to the DCCP performance over a long delay network. In this paper, our intention was to analyse DCCP/CCID4 performance in a more dynamic environment than what has been considered in previous work and to provide additional insight into how DCCP handles real VoIP traffic.

In our previous work, we have proposed a dynamic computation of the number of sent DCCP/CCID3 feedback messages as a function of the end-to-end connection delay [8]. This modification greatly improves the rate computation of DCCP/CCID3 over long delay links by increasing the responsiveness of TFRC. The latter is achieved by a more accurate and timely estimation of network parameters. In this previous work, we aimed at achieving a data rate comparable to TCP when sending or receiving a high rate data stream using CCID3. In this paper, we push further the idea of dynamic adjustments based on observed network conditions by investigating the parameters which will affect the perceived quality of VoIP carried by CCID4 over satellite links.

In the following section we present details of our experimental setup used to evaluate the VoIP performance with DCCP/CCID.

\section{EXPERIMENTAL SETUP}

Our experimental setup at the NICTA Laboratory in Sydney, Australia is presented in Figure 1. We use the IPSTAR satellite service, with the satellite modem connected to the remote site (client side) and through the IPSTAR satellite gateway to our gateway through the public Internet. For DCCP/CCID4, we use the experimental version of Linux kernel implementation (Aberdeen), which we have modified to include our proposed changes as described in Section V.

The VoIP application used is Asterisk Private Branch Exchange (PBX) [21], with voice codecs commonly used in IP telephony. We use G.711 [18], Speex [20], with and without discontinuous transmission (DTX) and G.729 [19]. The following table I lists details of the voice codecs used.

To have a fair comparison of quality with different codecs and different transport protocols, we use a pre-recoded sample of speech which is one side of a 10 min conversation. The analogue wave file is played into the VoIP PBX, encoded with 
Table I

VOICE CODEC PARAMETERS

\begin{tabular}{|c|c|c|c|}
\hline & $\begin{array}{c}\text { voice frame } \\
\text { (bits) }\end{array}$ & $\begin{array}{c}\text { silence frame } \\
\text { (bits) }\end{array}$ & $\begin{array}{c}\text { data rate } \\
\text { (kbits/s) }\end{array}$ \\
\hline G.711 & 1440 & N/A & 64 \\
G.729 & 160 & N/A & 8 \\
Speex & variable & N/A & variable \\
\hline
\end{tabular}

the appropriate voice codec, transmitted using UDP and the Inter Asterisk Exchange (IAX2) protocol [21] and captured by Wireshark [25]. Our stream replicator application reads the UDP/IP payload and reproduces the timing and packet sizes of the VoIP packets. This data stream is transmitted using DCCP and captured at the receiving end for analysis of packet loss and jitter. To produce examples of multiple conversations multiplexed into one data stream, we randomly start the pre-recorded conversation and apply the IAX2 multiplexing (trunking) option.

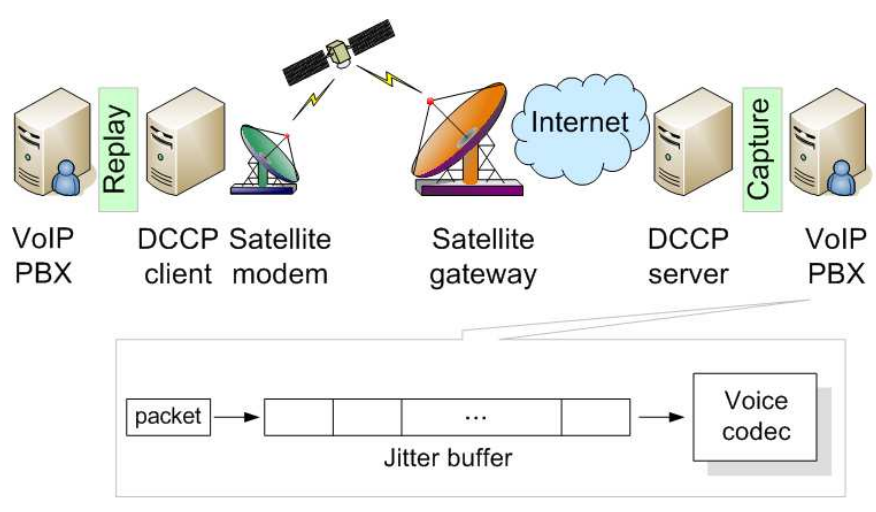

Figure 1. Experimental setup for live tests

Default DCCP/CCID4 parameters are used in all simulations, including the sender buffer size of five packets, consistent with other published work.

Previous experimental results [8] characterised the IPSTAR satellite network, which we consider a good representative of the growing number of IP based satellite services. IPSTAR uses shared access over radio channels and consequently can have both congestion and errors on the link. The network RTT and loss characterised during our long term experiments show, for small packet sizes, an average RTT of around $1000 \mathrm{msec}$ and a packet error rate (PER) of $0.1 \%$, consistent with published results which indicate an operating bit error rate (BER) of 107 [22]. Other satellite networks of interest would have similar or lower PER for packets of the same size, e.g. DVB-RCS [7] which has a specified BER of lower than 10-9.

The following section presents a summary of tests on IPSTAR performed for the DCCP/CCID4 and UDP protocol, the latter being currently most commonly used for VoIP applications.
Table II

AVERAGE PACKET ERROR RATE VALUES (\%) FOR DIFFERENT CODECS MEASURED ON IPSTAR LINK

\begin{tabular}{|c|c|c|c|}
\hline $\begin{array}{c}\text { Voice Codec } \\
\& \text { load }\end{array}$ & $\begin{array}{c}\text { Data rate } \\
(\mathrm{kbit} / \mathrm{s})\end{array}$ & $\begin{array}{c}\text { CCID4 (\%) } \\
(\%)\end{array}$ & $\begin{array}{c}\text { UDP } \\
(\%)\end{array}$ \\
\hline G.711 & 80 & 2.01 & 0.15 \\
G.729 & 44 & 1.24 & 0.1 \\
Speex & average & 1.84 & 0.1 \\
Speex/dtx & variable & 17.3 & 0.1 \\
Speex,5 calls & average & 2 & 0.15 \\
G.711,12 calls & 780 & 6.32 & 1.55 \\
\hline
\end{tabular}

Table III

AVERAGE PACKET ERROR RATE VALUES (\%) FOR DIFFERENT CODECS

\begin{tabular}{|c|c|c|}
\hline Jitter (ms) & avg & max \\
\hline UDP & 54 & 1000 \\
DCCP/CCID4 & 54 & 1000 \\
\hline
\end{tabular}

\section{TEST RESULTS AND PERFORMANCE ANALYSIS}

We have performed a number of experiments over the IPSTAR satellite network, for different voice codecs and under different load conditions. All experiments are of 10min duration. On IPSTAR, groups of experiments were performed at the same time to avoid differences in IPSTAR service load conditions impacting the test results.

We measure the packet loss rate and jitter values at the DCCP receiver, which we will use to calculate the VoIP quality in Section VI. We also monitor the various parameters which contribute to the DCCP/CCID4 sender rate, including RTT, loss event rate $p$ and receiver reported rate, to assist with analysis of the results. A summary of the results from 10 IPSTAR experiments, performed for the selected number of simultaneous calls, is presented in the following table II.

It can be observed that the packet loss with CCID4 has significantly higher values than UDP. Jitter values observed in the experiments are shown in the following table III

Our observations show that the main source of jitter is the network rather than the DCCP congestion control algorithm, i.e. the average and maximum jitter values do not noticeably differ between DCCP and UDP experiments.

The following section analyses the performance of DCCP in more detail and proposes an improvement to the algorithm.

\section{A. Performance Analysis}

To assist with analysis of the error rate results, we consider the potential sources of packet loss as perceived by the VoIP application. Packet loss rate at the input of the voice decoder can be broken down into the following contributing sources:

1) packet loss between the application and the transport protocol, resulting from the inability of the transport protocol rate control to provide adequate sending rate to the application;

2) packet loss on the link, which can be due to errors and/or congestion (closely related to the DCCP error event rate $p)$; 
3) the loss resulting from jitter, as the voice codec will consider all packets which arrive with incorrect timing as lost.

It is important to note that the packet loss between the application and the transport protocol will only be applicable to DCCP, as UDP does not have a specific sender rate and it just forwards application packets to the link.

For all experiments, in addition to capturing the transmitted and received data stream, we monitor the packets lost between the application and the DCCP sender and the DCCP reported losses. For the results summarised in Table II, only the experiments with G.711 had DCCP reported losses on the link and all other losses were between the application and DCCP sender. Therefore, it can be concluded that the majority of losses are caused by the inability of DCCP/CCID4 protocol to provide a high enough sending rate to the VoIP application. Additionally, as there are no reported losses, CCID4 is operating in slow start phase and never reaches the (expected and desired) congestion avoidance phase where the formula ensures TCP fairness.

The following section presents our proposals to modify the DCCP/CCID4 protocol in a way which will enable better handling of the VoIP application traffic while bearing in mid the requirements for fairness to competing TCP flows.

\section{IMPROVING DCCP/CCID4 FOR LONG DELAY LINKS}

By observing how the sender rate in calculated in CCID3/CCID4 phases described in Section II, it is intuitively clear that the VoIP packet losses will occur whenever there is transition from no speech to speech. Based on these observations and the experimental results presented in the previous sections, we propose to modify the CCID4 rate control in the two different ways.

In the first proposal, CCID4-N, we apply the existing CCID4 concept of replacing the measured packet size s by the equivalent packet size (of 1460 Bytes modified by the header correction factor) to the slow start phase.

Consequently, in slow start, the senders starting rate will remain one packet per second, but with the packet size modified according to our proposal. After the receiver feedback is available, the initial rate will be calculated by the following formula, which will replace formula 1 in rate calculations:

$$
X=\frac{4380}{R T T} \cdot \frac{s}{s+o h}
$$

The proposed modification will increase the starting rate and the initial rate, which will result in less packet loss in the initial stage of VoIP transmission and in transitions between silence and speech.

We also apply the CCID3 modification proposed in [8], so that $N$ feedback messages per RTT are used by the receiver in place of the default one feedback per RTT, when RTT is longer than a nominal value of e.g. $100 \mathrm{msec}$. This increases the speed of rate growth in slow start phase by applying formula 5 with increased frequency and, during the congestion control
Table IV

AVERAGE PACKET ERROR RATE VALUES (\%) FOR DIFFERENT CODECS MEASURED IN EXPERIMENTS ON IPSTAR LINK

\begin{tabular}{|c|c|c|c|}
\hline & $\begin{array}{c}\text { CCID4 } \\
(\%)\end{array}$ & $\begin{array}{c}\text { CCID4-N } \\
(\%)\end{array}$ & $\begin{array}{c}\text { CCID4-SCA } \\
(\%)\end{array}$ \\
\hline G.711 & 2.01 & 0.44 & 0.15 \\
G.729 & 1.24 & 0.08 & 0.1 \\
SPEEX & 1.84 & 0.15 & 0.1 \\
SPEEX/DTX & 17.3 & 0.16 & 0.1 \\
SPEEX/5calls & 2 & 0.34 & 0.15 \\
G.711/12calls & 6.32 & 3.76 & 1.5 \\
\hline
\end{tabular}

phase, provides more accurate values for changes of the RTT parameter used in formula 2 by more frequent measurements.

Our second proposal, CCID4-SCA, provides further optimisation for long delay links. We enhance the CCID4-N proposal by using a nominal value of RTT, e.g. $100 \mathrm{msec}$ in formula 6 , so the initial rate is provided by the following equation, which will replace formula 1 in DCCP rate calculations:

$$
X=4380 \cdot \frac{s}{\cdot} s+o h
$$

The proposed modification will further increase the initial rate for links with a delay longer than $100 \mathrm{msec}$, which should result in further reduction of packet loss in VoIP transmission in long delay links. As we only insert a fixed low RTT in calculating the initial rate, if the VoIP rate is too high for the potentially congested link, the standard CCID4 mechanism will detect and report errors and take DCCP into congestion control phase, which is where we want to operate in as this phase ensures long term fairness to other traffic sharing the link.

\section{A. Performance Evaluation}

The following table presents the summary of the packet error rate results from 10 IPSTAR experiments using the proposed modified CCID4 protocols, CCID4-N and CCID4SCA. For comparison purposes we also include the CCID4 results from Table II.

It can be observed that the packet error rate is significantly reduced by our proposed CCID4 modifications. Our proposal CCID4-N enables lower rate codecs and/or a lower number of multiplexed calls to perform with minimal loss rates. For higher number of calls producing data rates above the link rate, or in congested situations, our proposals will provide congestion control in the same way as CCID4.

In the following section we evaluate the VoIP quality for the different voice codecs, using the transport protocols and network load scenarios from our experiments.

\section{EVALUATION OF VOIP QUALITY}

Mean Opinion Score (MOS) is an ITU defined quality metrics for voice [15]. As MOS is a subjective measurement which cannot be easily applied to a variety of changing network conditions, ITU has also defined an objective evaluation methodology, the E-Model [16], which enables evaluation of the voice quality based on measured network parameters. The 
E-Models quality metrics is the $\mathrm{R}$ factor which can be used to calculate the MOS values as per [16].

We calculate the values of the R factor and MOS values using the overall packet loss rate as perceived by the voice codec at the receiving VoIP application, and measured delay. The results are shown for DCCP/CCID4, CCID4-N, CCID4SCA and UDP, which represents the baseline performance of voice codecs over the IPSTAR satellite network. To calculate the packet loss rate resulting from jitter in the received VoIP stream, we choose a buffer size of $400 \mathrm{msec}$, as a compromise between adding delay and loosing an increasing number of packets at the voice decoder. The jitter buffer size can be varied to further compensate for high jitter values, however that will not have an impact on the difference between the performance of DCCP and UDP as measured jitter values do not differ between those protocols.

Speex is not an ITU codec and does not have the defined parameter values necessary for calculating the $\mathrm{R}$ factor. For the purpose of estimating the performance of Speex, we roughly approximate the quality and error resilience of a Speex codec to the corresponding parameters for the G.729 codec. We consider this approximation sufficiently accurate for the purpose of this evaluation, as the reported MOS score for the Speex codec used in our experiments is 4.1 [20], comparable to the MOS value of 4.18 for the G.729 codec, resulting from the E-Model and MOS calculations under the same network conditions.

Figure 2 shows the $\mathrm{R}$ factor for selected codecs and numbers of calls on the IPSTAR satellite network for all the transport protocols considered. The value of advantage factor used in all $\mathrm{R}$ factor calculations is 30 .

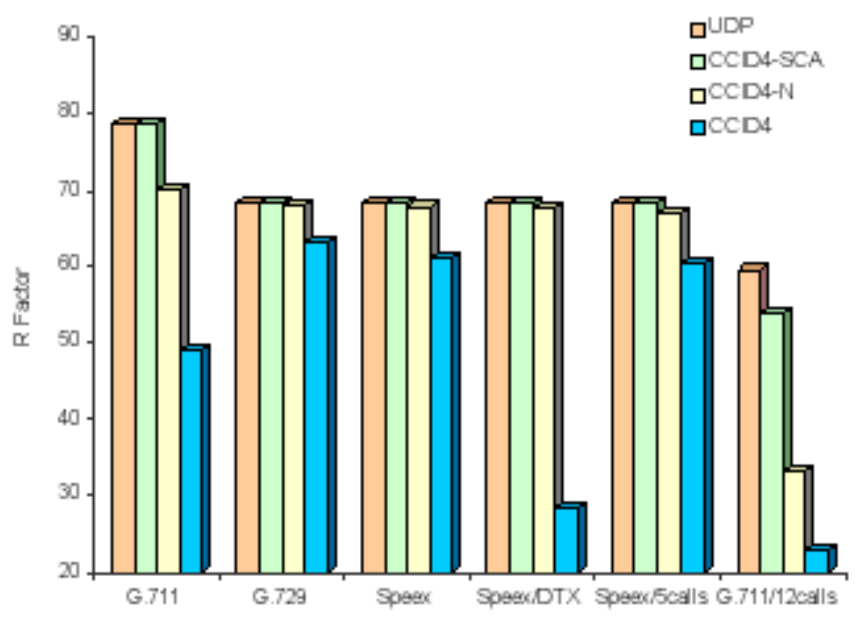

Figure 2. R factor for IPSTAR experiments, G.711, one and 12 calls, G.729, Speex, 1 and 5 calls, UDP, CCID4 and CCID4-N

To provide a clear view of the difference between the voice quality with UDP and with CCID4 variants, table V presents the degradation in MOS values compared to UDP.

MOS values of above 3.5 are considered acceptable for good quality It can be observed that the $\mathrm{R}$ factor values on
Table V

DEGRADATION OF MOS VALUES FROM UDP FOR DIFFERENT CONGESTION CONTROL MECHANISMS ON THE IPSTAR NETWORK

\begin{tabular}{|c|c|c|c|}
\hline & CCID4 & CCID4-N & CCID4-SCA \\
\hline G.711 & 0 & 0.36 & 1.45 \\
G.729 & 0 & 0.01 & 0.25 \\
SPEEX & 0 & 0.03 & 0.37 \\
SPEEX,DTX & 0 & 0.03 & 1.97 \\
SPEEX,5calls & 0 & 0.07 & 0.40 \\
G.711,12calls & 0.3 & 1.34 & 1.73 \\
\hline
\end{tabular}

Table VI

FAIRNESS INDEX VALUES FOR TWO FLOWS, TCP AND CCID4 VERSIONS

\begin{tabular}{|c|c|c|c|c|}
\hline Fairness Index & CCID4 & CCID4-N & CCID4-SCA & UDP \\
\hline TCP+G.711/12calls & 0.9997 & 0.99997 & 0.99997 & 0.74 \\
TCP+1Mbit/s data rate & 0.985 & 0.98 & 0.98 & 0.5 \\
\hline
\end{tabular}

IPSTAR network range between an acceptable 79 (with a corresponding to a MOS value of 3.9) for a G.711 call with either UDP or CCID4-SCA to unacecptable values values of around 60 for Speex/DTX and more surprisingly for G.711. This is due to the different contribution of error rate to voice codec quality i.e. G.711 is less resilient to errors than G.729 and it also has more errors with CCID4 as the higher rate change in the early part of the conversation results in more losses.

Our proposals improves the MOS values ...

Overall, there will be a cost to using a transport protocol which has rate control and ensures fairness to other competing flows and we believe the changes proposed in this paper minimise this cost.

In the following section, we will evaluate the fairness of the proposed DCCP/CCID4 modifications to competing TCP flows.

\section{FAIRNESS TO OTHER FLOWS}

To evaluate fairness of multiple flows, we use Jains fairness index [23].

As VoIP traffic is limited by the application, farness can only be considered for VoIP streams which result from a number of parallel (multiplexed) calls which would require unfair capacity when sharing the link with other flows. We compare fairness to TCP of a VoIP data stream resulting from 12 parallel G.711 calls (with a data rate higher than half the nominal link rate on the IPSTAR equivalent link). We also show the fairness to TCP of a flow with rate equivalent to the nominal link rate on the IPSTAR link To illustrate the advantage of using DCCP, we also present the fairness results for UDP. The results of the fairness test are presented in the following table VI.

It can be observed that both DCCP and DCCP-N are equally fair to a TCP flow and that UDP, as expected, takes all the bandwidth it requires regardless of other flows.

The following section presents our conclusions and ideas for future work. 


\section{CONCLUSIONS AND FUTURE WORK}

We have evaluated the performance of DCCP/CCID4 on a live satellite link and proposed improvements which result in no loss of fairness to TCP traffic compared to the original CCID4, with both being fair. We have demonstrated the advantage of using DCCP over UDP when the network is shared with other TCP traffic flows as is the case in the vast majority of scenarios where VoIP is present, by evaluating a number of cases which include different voice codecs and different number of multiplexed simultaneous VoIP calls.

The main issue identified with using CCID4 for VoIP. Firstly, in majority of the cases, due to low VoIP application rate, CCID4 only operates in the slow start phase and does not use the congestion avoidance mechanism. Secondly, in periods of transition from silence to speech, depending on the rate required by the VoIP application, in most cases CCID4 cannot support the required application rate and this results in significant packet loss, particularly on long delay links.

Our proposed modifications significantly reduce the packet loss between the application and DCCP sender in a long delay environment. They could also provide similar benefits in networks which have a lower delay, in enabling a faster slow start, higher minimum rate and a more accurate parameter measurement resulting in a more responsive rate adjustment which better matches the varying network conditions. Both proposals require minimal changes to CCID4 specification and no interworking with other network components.

We would also like to point out another advantage which DCCP can provide over UDP for VoIP, in addition to fairness. DCCP awareness of transport layer losses can be used for call admission control in VoIP applications. As the number of calls increases and reaches the level where packets are lost, the DCCP measured loss rate can be used to block further calls being allowed by the VoIP application.

We plan to extend the evaluation of DCCP to long range wireless links like WiMax and consider further improvements to CCID4 and CCID3 algorithms.

\section{ACKNOWLEDGEMENT}

This research work has been supported by funding from National ICT Australia (NICTA). Authors would also like to thank IPSTAR Australia for their continuing support.

\section{REFERENCES}

[1] E. Kohler, M. Handley and S. Floyd, Datagram Congestion Control Protocol (DCCP), IETF, Request For Comments 4340, Mar. 2006.

[2] S. Floyd, E. Kohler and J. Pahdye, Profile for Datagram Congestion Control Protocol (DCCP) Congestion Control ID 3: TCP-Friendly Rate Control (TFRC), IETF, Request For Comments 4342, Mar. 2006.

[3] M. Handley, S. Floyd, J. Pahdye, and J. Widmer, TCP Friendly Rate Control (TFRC): Protocol Specification, IETF, Request For Comments 3448, Jan. 2003.

[4] S. Floyd and E. Kohler, Profile for Datagram Congestion Control Protocol (DCCP) Congestion Control ID 4: the Small-Packet Variant of TFRC Congestion Control, IETFWork, draft, Jun. 2006.

[5] WildBlue, http://wildblue.com.

[6] IPSTAR, http://ipstar.com

[7] ETSI EN, Digital Video Broadcasting (DVB); Interaction channel for satellite distribution systems, Tech. Rep. 301790 V1.4.1, Sep. 2005.
[8] G.Sarwar, R.Boreli, E.Lochin and G.Jourjon Experimental performance of DCCP over live satellite and long range wireless links, to be presented at IWSSC 2008, Toulouse, Oct. 2008.

[9] H. Balan, L. Eggert, S. Niccolini and M. Brunner, An Experimental Evaluation of Voice Quality over the Datagram Congestion Control Protocol, INFOCOM 2006

[10] A. Sathiaseelan and G. Fairhurst, Performance of VoIP using DCCP over a DVB-RCS Satellite Network, in Proc. of IEEE ICC 07, Glasgow, Jun. 2007.

[11] , Use of Quickstart for Improving the Performance of TFRC-SP Over Satellite Networks, in Proc. of the International Workshop on Satellite and Space Communications (IWSSC2006), Leganes, Sep. 2006.

[12] S. Floyd, M. Allman and A. Jain, Quick-Start for TCP and IP,IETFWork, draft, 2006.

[13] E. Kohler and S. Floyd, Faster Restart for TCP Friendly Rate Control (TFRC), IETFWork, draft, Jun. 2006.

[14] Waikato University, DCCP site

[15] ITU TRECP.800, Methods for subjective determination of transmission quality

[16] ITU T-REC-G.107-200503-I, The E-Model, a computational model for use in transmission planning

[17] ITU TRECG.113200711I, Transmission impairments due to speech processing

[18] ITU T-REC-G.711-198811-I, Pulse code modulation of voice frequencies

[19] ITU T-REC-G.729, Coding of speech at $8 \mathrm{kbit} / \mathrm{s}$ using conjugatestructure algebraic-code-excited linear prediction (CS-ACELP)

[20] Speex codec, www.speex.org

[21] Asterisk PBX, www.voip.org

[22] W. Thesling, M. Vanderaar, M. Thompson, P. Hamilton, P. Panuwattanawong and R. Gedney, Two Way Internet over IPSTAR Using Advanced Error Correctiona and Dynamic Links, in Proc. of 20th AIAA International Communication Satellite Systems Conference, AIAA-2002-1944, May 2002

[23] R. Jain, The Art of Computer Systems Performance Analysis: Techniques for Experimental Design, Measurement, Simulation, and Modeling, Wiley- Interscience, New York, NY, April 1991, ISBN:0471503361

[24] Bertsekas and Gallager, Data networks, Prentice Hall 1992

[25] www.wireshark.org 